\title{
Neuromuscular electrical stimulation improves exercise capacity in adult patients with chronic lung disease: a meta-analysis of English studies
}

\author{
Haihong Gong ${ }^{1}$, Qinghe Jiang ${ }^{2}$, Dongchao Shen ${ }^{3}$, Jinming Gao ${ }^{1}$ \\ ${ }^{1}$ Department of Respiratory Disease, Peking Union Medical College Hospital, Chinese Academy of Medical Sciences and Peking Union Medical \\ College, Beijing 100730, China; ${ }^{2}$ Department of Respiratory Disease, Liaocheng People's Hospital, Liaocheng 252000, China; ${ }^{3}$ Department of \\ Neurology, Peking Union Medical College Hospital, Chinese Academy of Medical Sciences and Peking Union Medical College, Beijing 100730, \\ China \\ Contributions: (I) Conception and design: J Gao; (II) Administrative support: D Shen; (III) Provision of study materials or patients: H Gong, Q Jiang; \\ (IV) Collection and assembly of data: H Gong, Q Jiang; (V) Data analysis and interpretation: H Gong, Q Jiang; (VI) Manuscript writing: All authors; \\ (VII) Final approval of manuscript: All authors. \\ Correspondence to: Jinming Gao, MD. Professor of Medicine, Department of Respiratory Diseases, Peking Union Medical College, \#1 Shuaifuyuan, \\ Dongcheng District, Beijing 100730, China. Email: gjinming@yahoo.com.
}

Background: Neuromuscular electrical stimulation (NMES) has been suggested as an alternative rehabilitative therapy to enhance exercise performance and skeletal muscle function in adult patients with chronic lung disease. However, the results of individual studies have been inconsistent. We performed a meta-analysis to evaluate the effectiveness of NMES with regard to increasing exercise capacity, quadriceps strength, muscle mass, cross-sectional area, and quality of life and decreasing dyspnea in adult patients with chronic lung disease.

Methods: A systematic search was conducted of the PubMed, Cochrane Library and EMBASE databases for randomized controlled trials (RCTs) published in English-language journals before January 2018. Data were extracted using standardized forms, and the weighted mean difference (WMD) or standardized mean difference (SMD) with 95\% confidence intervals (CIs) was calculated.

Results: Eleven RCTs involving 368 patients were included in this meta-analysis. The pooled results showed that NMES significantly improved the 6-min walk distance (WMD: $37.93 \mathrm{~m}, 95 \%$ CI: 19.53-56.33 m; $\mathrm{P}<0.0001$; $\mathrm{P}$ for heterogeneity $=0.11 ; \mathrm{I}^{2}=47 \%$ ) but not the incremental shuttle walk test (WMD: $18.18 \mathrm{~m}$, 95\% CI: -79.41 to $115.77 \mathrm{~m}, \mathrm{P}=0.72$; P for heterogeneity $<0.0001, \mathrm{I}^{2}=94 \%$ ) or endurance shuttle walk test (ESWT) (WMD: $96.73 \mathrm{~m}, 95 \% \mathrm{CI}:-45.58$ to $239.03 \mathrm{~m}, \mathrm{P}=0.18$; P heterogeneity $=0.22, \mathrm{I}^{2}=34 \%$ ). Moreover, NMES was associated with a significant improvement in quadriceps strength (SMD: 1.14, 95\% CI: 0.86-1.43, $\mathrm{P}<0.00001$; P heterogeneity $=0.02, \mathrm{I}^{2}=58 \%$ ).

Conclusions: This systemic review and meta-analysis provided evidence supporting the beneficial role of NMES in improving exercise capacity in patients with chronic respiratory disease.

Keywords: Chronic lung disease; electrical stimulation; neuromuscular; meta-analysis; exercise performance; quadriceps

Submitted Jul 05, 2018. Accepted for publication Nov 12, 2018.

doi: $10.21037 /$ jtd.2018.11.133

View this article at: http://dx.doi.org/10.21037/jtd.2018.11.133 


\section{Introduction}

Chronic lung disease is one of the leading causes of morbidity and mortality worldwide and frequently results in exercise intolerance and peripheral muscle dysfunction that have been recognized as extrapulmonary involvement (1-3). Reduced exercise capacity and quadriceps weakness have adverse impacts on lung function and mortality (2-4). Pulmonary rehabilitation, including aerobic exercise and resistance training, can improve muscle function and the related clinical consequences $(5,6)$. However, some adult patients with advanced progressive disease are unwilling or unable to perform whole-body exercise because of the high symptom burden or breathlessness, even at low levels of exertion.

Neuromuscular electrical stimulation (NMES) has been introduced as an alternative treatment to enhance lower limb muscle strength in healthy subjects (7) and is well tolerated by patients as a means of improving exercise capacity $(8,9)$ and muscle function (10). However, these studies lack power and precision because of their small sample sizes, varying outcome measures, and inconclusive results $(11,12)$. This meta-analysis aimed to assess the effect of NMES on exercise capacity, quadriceps strength and other clinical outcomes in adult patients with chronic lung disease.

\section{Methods}

\section{Data sources and search strategy}

This systematic review was conducted in accordance with the guidelines in the handbook of the Centre for Reviews and Dissemination. A computerized literature search was performed in the following databases up to January 2018: Medline/PubMed, EMBASE and the Cochrane Library. The following keywords were used: NMES and lung. The broad inclusion criteria for eligible articles were as follows: (I) population: patients with chronic lung disease; (II) intervention: NMES of the lower limbs, alone or in combination with other exercise programs; (III) comparison: NMES $v s$. any treatment including sham exercise programs or no treatment; and (IV) study design: randomized clinical trials. Articles in a language other than English, reviews, notes, editorials, qualitative studies, and congress abstracts were excluded.

\section{Data extraction}

Two independent reviewers (Gong and Shen) separately extracted the data according to the inclusion criteria. Any disagreements between the reviewers were usually resolved by consensus. Discrepancies were resolved by a third independent reviewer (Jiang). Analytical data missing from the primary reports were requested from the authors. When the same population was reported in several publications, we only included the most informative article or the most complete study to avoid the duplication of information.

\section{Types of outcome measures}

\section{Primary outcome}

The primary outcome measure was exercise performance, which was mainly measured by the 6 min walk distance (6MWD), incremental shuttle walk test (ISWT), and endurance shuttle walk test (ESWT).

\section{Secondary outcomes}

(I) Quadriceps muscle strength was evaluated using an isokinetic strength test (peak torque) or maximal voluntary contraction following NMES.

(II) Muscle mass or cross-sectional area was measured.

(III) Health-related quality of life was measured.

(IV) Dyspnea was evaluated by the Borg scale daily or during or immediately after exercise.

\section{Quality assessment and risk of bias assessment}

The quality of the included studies was assessed using the Jadad scale (13). A score $\leq 2$ indicated low quality, and a score $\geq 3$ indicated high quality (14). This study was conducted in accordance with the Preferred Reporting Items for Systematic Reviews and Meta-Analyses (PRISMA) statement (15).

\section{Statistical analysis}

Revman5.1.0 (http://imscochrane.org/revman) was used to perform the meta-analysis. Differences were calculated as weighted mean differences (WMDs) or standardized mean differences (SMDs) with 95\% confidence intervals (CI) for continuous outcomes. All measures were pooled across studies using a random effects model. Heterogeneity across studies was tested using the $\mathrm{I}^{2}$ statistic. Studies with $\mathrm{I}^{2}$ statistics of $25-50 \%$ were considered to have low heterogeneity, those with $\mathrm{I}^{2}$ statistics of $50-75 \%$ were considered to have moderate heterogeneity, and those with $\mathrm{I}^{2}$ statistics $>75 \%$ were considered to have a high degree 


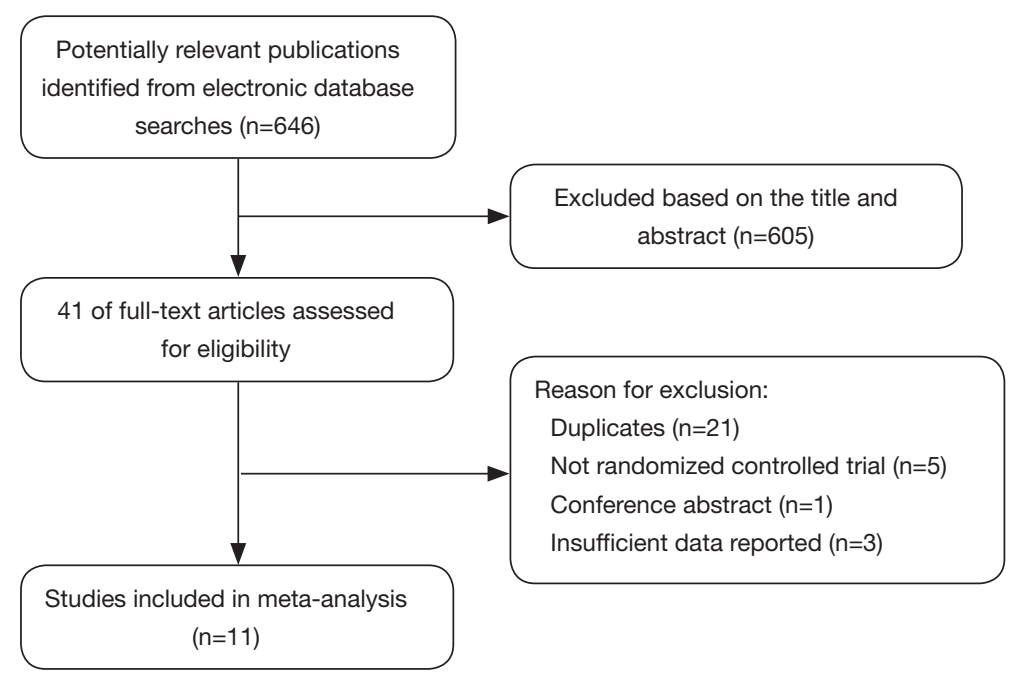

Figure 1 Search strategy and flow of participants in the meta-analysis.

of heterogeneity (16). If $\mathrm{I}^{2}$ was $>50 \%$, a random effects model was used due to the higher degree of heterogeneity. Potential sources of heterogeneity were identified by sensitivity analyses conducted by omitting each study in turn and investigating the influence of each study on the overall pooled estimate. A subgroup analysis was also conducted based on different measurement indicators. Publication bias was not assessed because of the limited number $(<10)$ of studies included in each analysis. $\mathrm{P}<0.05$ was considered significant.

\section{Results}

\section{Bibliographic search results}

The initial search yielded 646 relevant articles. In total, 605 studies were excluded based on the titles and abstracts for various reasons (reviews, nonrandomized studies, or not relevant to our analysis). In total, 41 studies were identified for full-text analysis. Following further analysis of the selected studies' adherence to the inclusion criteria, 11 RCTs were selected for this meta-analysis, and 30 studies were excluded from the final analyses. Figure 1 shows the different phases of the search process.

\section{Study characteristics}

The main characteristics of the 11 RCTs, of which 8 tested participants with COPD, 2 tested participants with non- small cell lung cancer and 1 tested participants with cystic fibrosis (CF), included in the meta-analysis are presented in Table 1. The included studies were published between 2002 and December 14, 2015. The sample size of the trials ranged from 14 to 120 (a total of 368, with 216 males and 152 females). The patients ranged in age from 28 to 70 years old. Follow-up periods ranged from 4 to 11 weeks. Five RCTs $(9,21,23-25)$ reported 6 MWDs and were pooled in the meta-analysis. Two studies provided data for the ISWT $(17,26)$ and three studies provided data for the ESWT $(18,22,26)$. All RCTs reported quadriceps muscle strength, but only eight provided the data (mean \pm standard deviation or standard error). Among those eight studies, five RCTs reported isokinetic peak torque $(17,18,20,21,24)$, while the other three RCTs used other measures of force (e.g., in kilograms) $(9,19,23)$. Two RCTs reported muscle mass $(21,25)$ and CSA $(9,22)$. A total of five studies reported dyspnea $(21-24,26)$ and provided data on the patients' quality of life $(9,19,24-26)$.

\section{Quality assessment and risk of bias assessment}

Two investigators (Gong and Shen) agreed on every item of the Jadad score. The mean Jadad score was 3.6 (standard deviation $=0.65$ ). The risk of bias analysis showed that only three RCTs adequately reported the randomization protocol used $(9,24,26)$, while five RCTs $(9,20,24-26)$ described the method used to conceal the allocation of patients to treatments (Figure 2). 


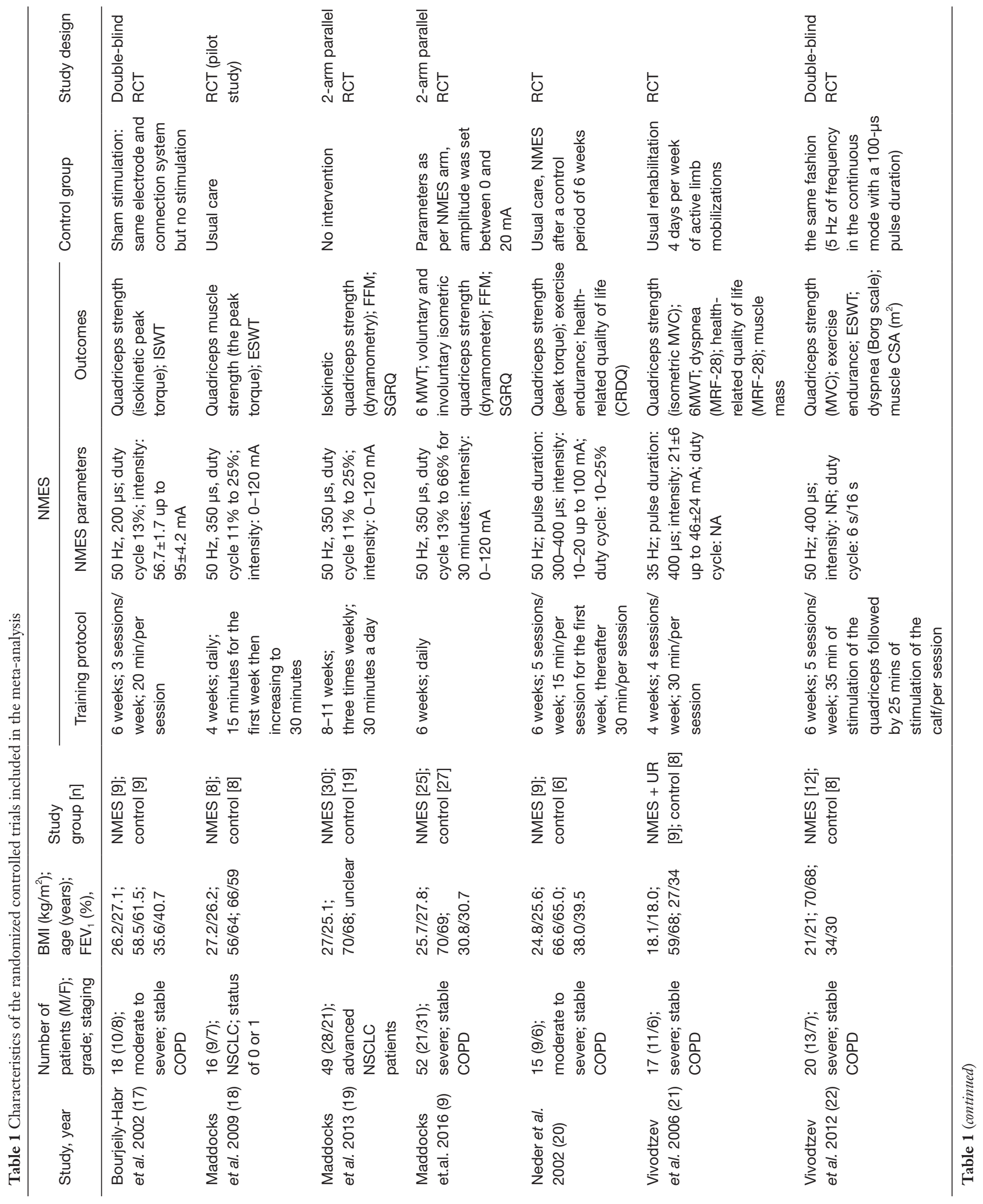




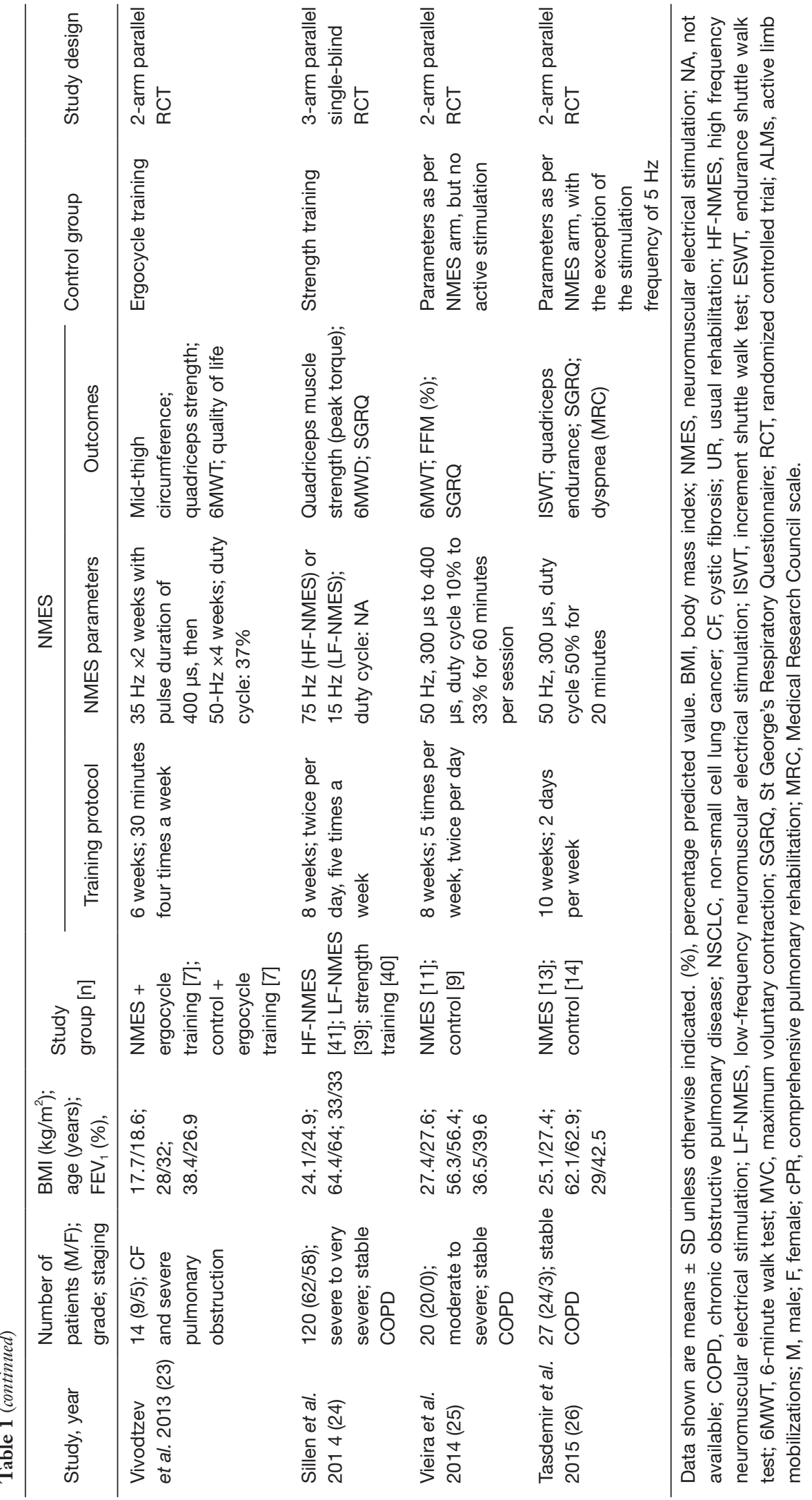


A

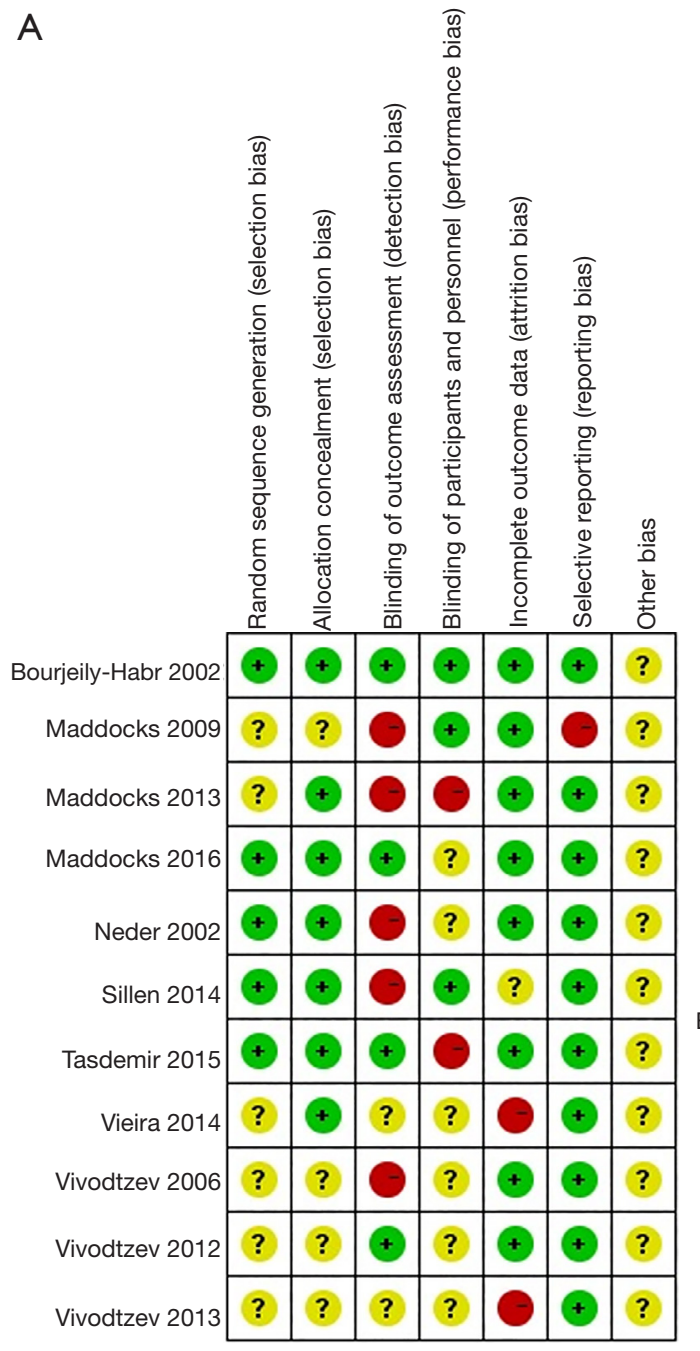
B

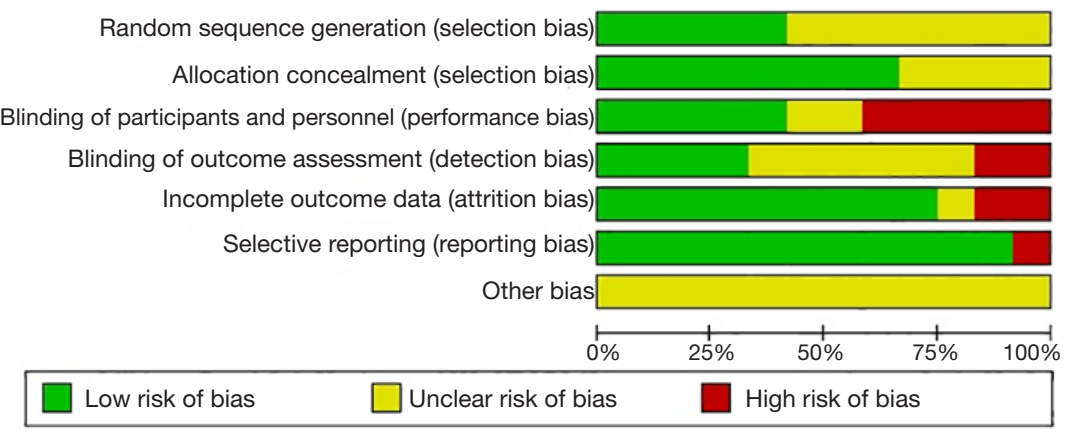

Figure 2 Risk of bias analysis. (A) Risk of bias summary: judgments regarding each risk of bias item for each included RCT; (B) risk of bias graph: judgments regarding each risk of bias item presented as percentages across all included RCTs. RCTs, randomized controlled trials.

\section{Meta-analysis of outcome measures}

\section{Primary outcomes: exercise capacity}

The aggregated results from five studies $(9,21,23-25)$ suggested that NMES was associated with the improvement of the 6MWD (WMD 37.93 m; 95\% CI: 19.53-56.33 m; P<0.0001; $\mathrm{P}$ for heterogeneity $=0.11 ; \mathrm{I}^{2}=47 \%$ ) (Figure 3). Two studies used the ISWT $(17,26)$ as an outcome measure, and three studies $(18,22,26)$ reported the ESWT. We failed to find statistically significant differences in the ISWT or ESWT between the NMES treatment group and the control group [ISWT $18.18 \mathrm{~m}$ (95\% CI: -79.41 to $115.77 \mathrm{~m}$; $\mathrm{P}=0.72$, P heterogeneity $<0.0001, \mathrm{I}^{2}=94 \%$ ); ESWT (WMD 96.73 m; $95 \%$ CI: -45.58 to $239.03 \mathrm{~m}$; $\mathrm{P}=0.18$, P heterogeneity
$=0.22, \mathrm{I}^{2}=34 \%$ )] (Figure 3). We did not perform sensitivity analyses because only two RCTs were included.

\section{Secondary outcomes \\ Quadriceps muscle strength}

Eight RCTs $(9,17-21,23,24)$ reported quadriceps muscle strength, and these data were pooled in the current metaanalysis. Considerable heterogeneity existed among the included studies $\left(\mathrm{I}^{2}=58 \%\right)$, and we used a random effects model for the pooled analysis. The aggregate results suggested that NMES was associated with a significant improvement in quadriceps strength (SMD: 1.14; 95\% CI: 0.86-1.43; $\mathrm{P}<0.00001$ ) (Figure 4). Removing the study by Sillen et al. [2014] (24), in which NMES was compared to 


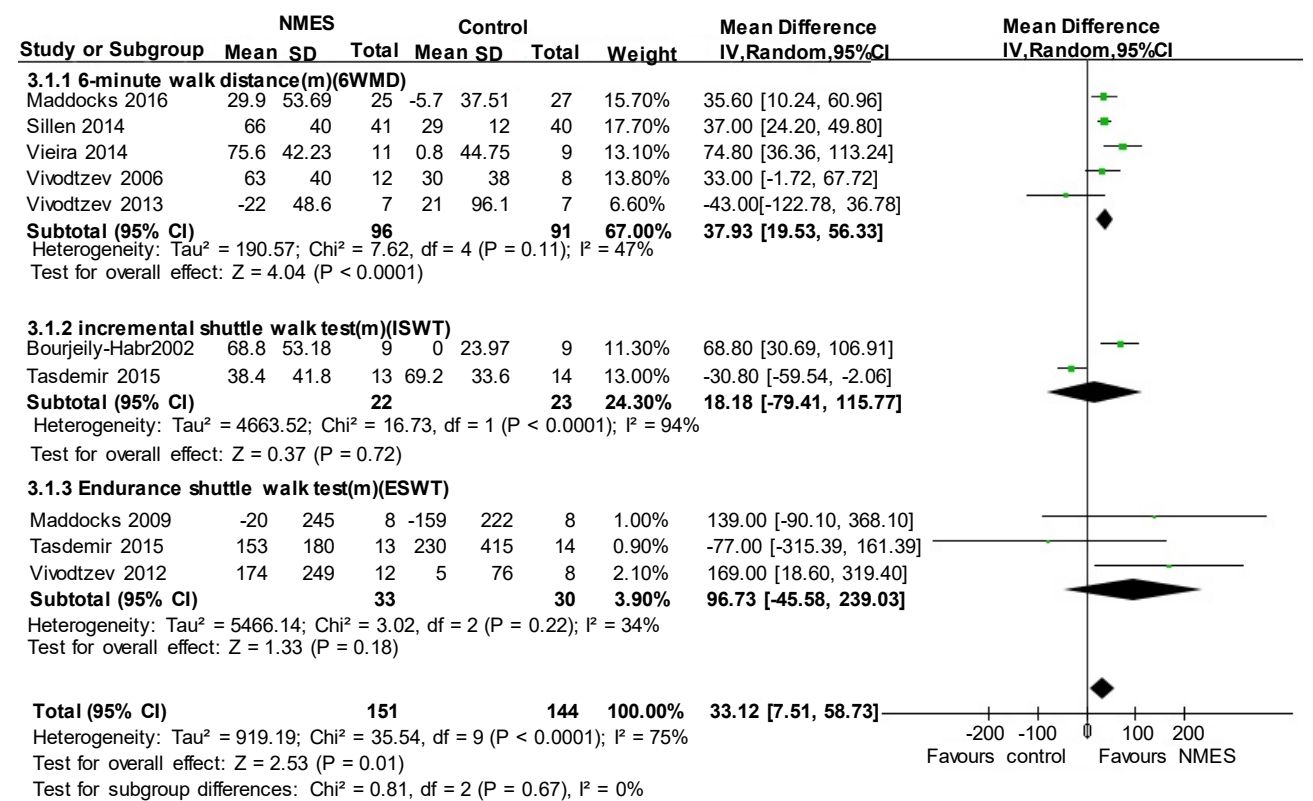

Figure 3 A forest plot of the meta-analysis of RCT comparing NMES with the control group for the change in exercise performance as analyzed by the random effects model. Each block represents a study, and the area of each block is proportional to the precision of the mean treatment effect in that study. The horizontal line represents the $95 \%$ confidence interval (CI) for the treatment effect. The center of the diamond is the average treatment effect across studies, and the width of the diamond denotes its $95 \%$ CI. RCT, randomized controlled trial; NMES, neuromuscular electrical stimulation; 6MWD, 6-min walking distance; ISWT, increment shuttle walk test; ESWT, endurance shuttle walk test.

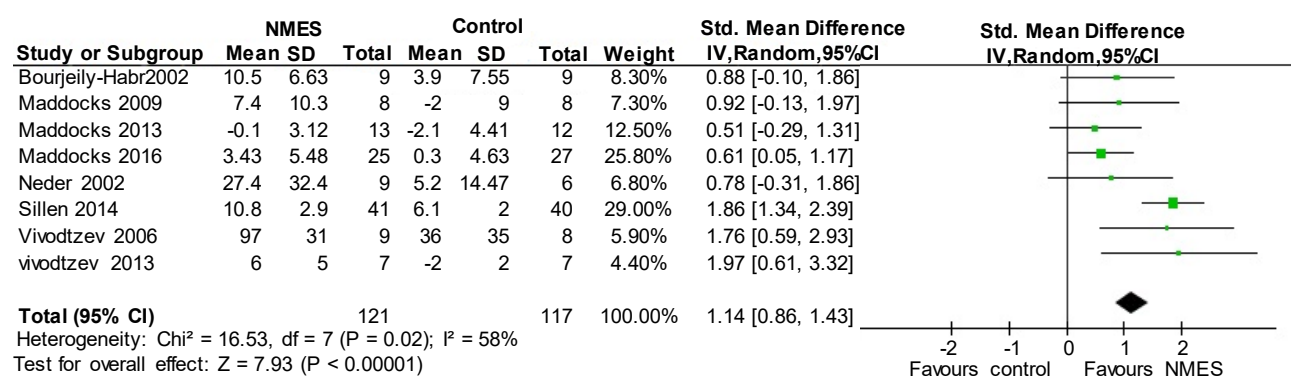

Figure 4 Meta-analysis of RCTs evaluating the effects of NMES on quadriceps muscle strength by the random effects model. RCT, randomized controlled trial; NMES, neuromuscular electrical stimulation.

resistance training, significantly decreased the heterogeneity $\left(\mathrm{I}^{2}=6 \%\right)$ and the point estimate for effectiveness (SMD: 0.85, 95\% CI: 0.51-1.19).

\section{Muscle mass and CSA}

Two RCTs reported muscle mass $(21,25)$ and CSA $(9,22)$, and these data were pooled in the current study. The pooled results revealed that NMES was associated with an increase in muscle mass (SMD 0.95; 95\% CI: 0.25-1.64; P=0.008; P for heterogeneity $=0.37 ; \mathrm{I}^{2}=0 \%$ ) and CSA (SMD 1.08; 95\%
CI: 0.56-1.59; $\mathrm{P}<0.0001 ; \mathrm{P}$ for heterogeneity $=0.01 ; \mathrm{I}^{2}=0.83$ ) (Figure 5). The heterogeneity test was significant for CSA. We did not perform sensitivity analyses because only two RCTs were included.

\section{Health-related quality of life}

Five studies $(9,19,24-26)$ reported health-related quality of life (SGRQ) following NMES. The overall mean difference (MD) for NMES compared to the control was -1.53 (95\% CI: -7.54 to $4.48 ; \mathrm{P}=0.62$; $\mathrm{P}$ for heterogeneity 


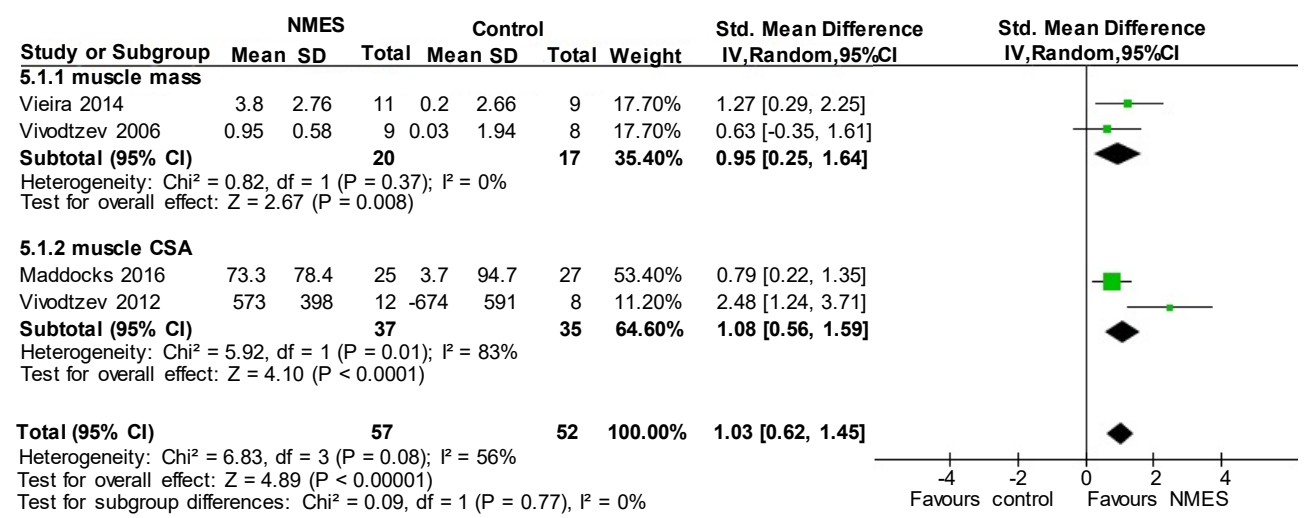

Figure 5 Meta-analysis of RCTs evaluating the effects of NMES on muscle mass and CSA by the random effects model. RCT, randomized controlled trial; NMES, neuromuscular electrical stimulation. CSA, cross-sectional area.

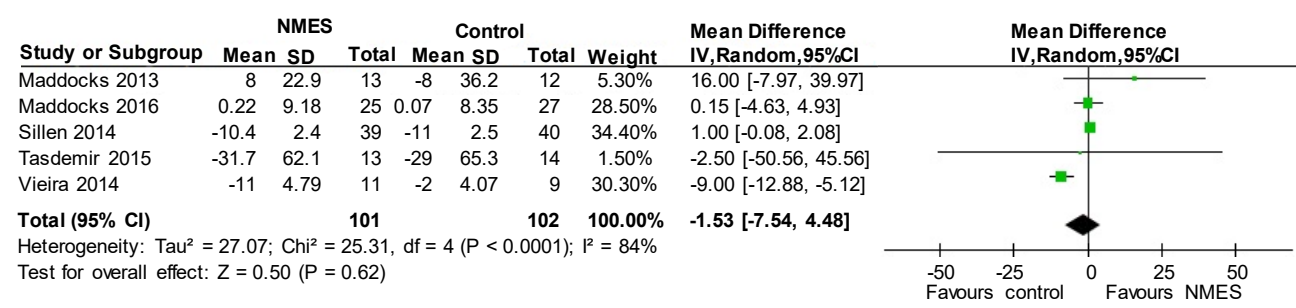

Figure 6 A forest plot of meta-analysis of RCTs comparing NMES with the control group for the change in the quality of life as analyzed by the random effects model. RCT, randomized controlled trial; NMES, neuromuscular electrical stimulation.

$<0.0001 ; \mathrm{I}^{2}=84 \%$ ) (Figure 6 ). When the RCT by Vieira et al. [2014] (25), the only RCT in which the total SGRQ score improved in the NMES group, was omitted, the value of the WMD for health-related quality of life was $0.99(95 \%$ CI: -0.07 to 2.04; $\mathrm{P}=0.07$; $\mathrm{P}$ heterogeneity $=0.65, \mathrm{I}^{2}=0 \%$ ).

\section{Breathlessness}

Two studies $(21,24)$ reported breathlessness in daily life, and four studies $(21-23,26)$ reported breathlessness using the Borg scale during exercise following NMES. The pooled results suggested that NMES was not associated with a significantly reduced dyspnea score in daily life (WMD $-0.70 ; 95 \%$ CI: -2.05 to $0.66 ; \mathrm{P}=0.31$; $\mathrm{P}$ for heterogeneity $\left.=0.010 ; \mathrm{I}^{2}=85 \%\right)$ or during exercise (WMD $-0.62 ; 95 \% \mathrm{CI}$ : -1.66 to $0.42 ; \mathrm{P}=0.24 ; \mathrm{P}$ for heterogeneity $=0.29 ; \mathrm{I}^{2}=20 \%$ ) (Figure 7).

\section{Discussion}

This current systematic review compiled evidence from a large number of RCTs and assessed the effectiveness of NMES in adult patients with chronic lung disease. Our principal finding is that NMES significantly improves exercise capacity and quadriceps muscle strength, which suggests that NMES has positive effects in adult patients with chronic lung disease. More high-quality RCTs, with low risk of bias and adequate sample sizes, are required to confirm its effects.

Although heterogeneity existed among the pooled studies, we can draw some conclusions from this systematic review. Our results showed that NMES improved exercise performance (6MWD: WMD 37.93 m; 95\% CI: 19.53$56.33 \mathrm{~m}$ ) and quadriceps muscle strength (SMD: 1.14; 95\% CI: 0.86-1.43) compared with the control group. Inconsistent with this improvement in muscle strength following NMES, this updated review indicated that no significant differences were found between NMES and the control group in terms of muscle mass, CSA, dyspnea and SGRQ. Breathlessness and SGRQ consistently improve with pulmonary rehabilitation $(27,28)$. The discordance between the overall improvement in muscle and exercise capacity after NMES and the lack of improvement in patient-reported outcomes including breathlessness and 


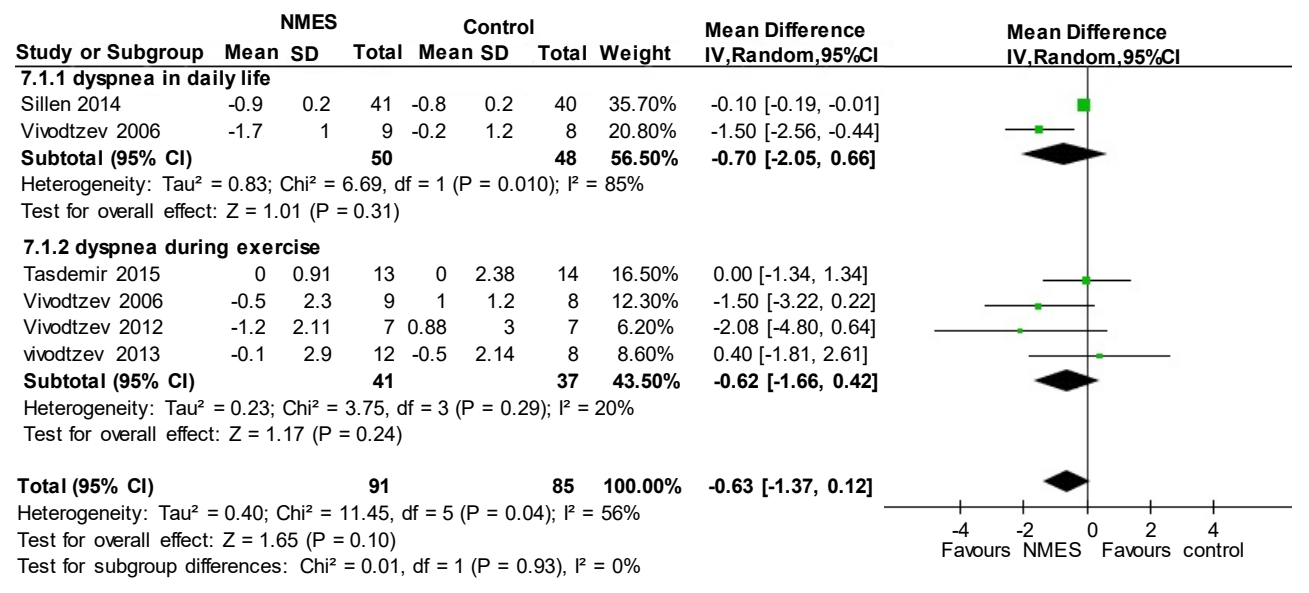

Figure 7 Meta-analysis results summarizing the effects of NMES on dyspnea as analyzed by the random effects model. RCT, randomized controlled trial; NMES, neuromuscular electrical stimulation.

SGRQ may be explained as follows: first, patient-reported outcomes are influenced by many other factors (29); second, extended follow-up might demonstrate a beneficial effect of NMES on breathlessness and SGRQ (28); and third, because of the limited number of high-quality RCTs, further investigation is needed to demonstrate the true effect of NMES.

When interpreting clinical measures, it is necessary to compare the results with minimal clinically important differences (MCID). The change in $6 \mathrm{MWD}$ was higher than the latest MCID ( $\geq 26 \mathrm{~m}$ ) (30). Unfortunately, the clinical relevance of this change in quadriceps strength and other clinical outcomes is unclear (31).

Our findings corroborate the results of three previous systematic reviews about the use of NMES in COPD or chronic heart failure patients. Vivodtzev 2008 (32) examined five RCTs conducted with people with COPD, and Sillen 2009 (33) included a total of 14 studies (9 with patients with chronic heart failure; 5 with patients with COPD) and revealed that NMES improved skeletal muscle function and exercise capacity, which was in accordance with our results. Compared with the other recent review (34), the major strengths of our meta-analysis are as follows: first, a larger number of pooled studies and participants were included, and the target population was not the same. Second, more outcome endpoints (dyspnea, muscle mass and CSA) were reported. Third, we used exercise performance as the primary outcome. However, Pan 2014 (12) concluded that evidence to support the positive effect of NMES on the quadriceps strength in patients with COPD was not adequate. The meta-analysis by Pan included only two studies and four studies evaluating the effects of NMES on exercise capacity and skeletal muscle function, respectively, which may be a possible cause of these inconsistent results.

Several limitations of our meta-analysis should be mentioned. First, some studies did not report the main endpoint, and more high-quality studies are needed to improve the reliability of the results. Second, the pooled estimate effects for quadriceps muscle strength have significant heterogeneity because some clinical differences among the participants existed and the intervention protocols were not the same. Third, regarding patient selection, only three articles that did not evaluate COPD (one investigating patients with $\mathrm{CF}$ and two investigating patients with lung cancer) were included in the metaanalysis, which, to a certain extent, may compromise the external validity. Fourth, the exercise protocols were not all the same, and the assessment of dyspnea should be considered with great care. Finally, the language of the pooled studies was limited to English, and missing data may be a possible source of publication bias.

Based on the current results, the present study provides evidence regarding the effectiveness of NMES for pulmonary rehabilitation. First, NMES protocols varied widely, and future studies should move beyond testing methodological standards (for example, optimal NMES protocol and the dosage of NMES). Second, long-term longitudinal follow-up data are needed to better understand the effects of NMES. Third, understanding the detailed biochemical mechanisms underlying the functional improvements following NMES requires further investigation. 


\section{Conclusions}

In summary, the current meta-analysis showed that NMES was beneficial for the management of patients with chronic lung disease because it improves physical activity and lower limb muscle function. More multicenter RCTs with large sample sizes and longer follow-up periods are encouraged to further confirm this conclusion and to investigate the impact of NMES on patients with chronic respiratory disease.

\section{Acknowledgements}

None.

\section{Footnote}

Conflicts of Interest: The authors have no conflicts of interest to declare.

\section{References}

1. Swallow EB, Reyes D, Hopkinson NS, et al. Quadriceps strength predicts mortality in patients with moderate to severe chronic obstructive pulmonary disease. Thorax 2007;62:115-20.

2. Seymour JM, Spruit MA, Hopkinson NS, et al. The prevalence of quadriceps weakness in COPD and the relationship with disease severity. Eur Respir J 2010;36:81-8.

3. Barreiro E, Sznajder JI, Nader GA, et al. Muscle dysfunction in patients with lung diseases: a growing epidemic. Am J Respir Crit Care Med 2015;191:616-9.

4. Jones SE, Maddocks M, Kon SS, et al. Sarcopenia in COPD: prevalence, clinical correlates and response to pulmonary rehabilitation. Thorax 2015;70:213-8.

5. Nyberg A, Carvalho J, Bui KL, et al. Adaptations in limb muscle function following pulmonary rehabilitation in patients with COPD - a review. Rev Port Pneumol (2006) 2016;22:342-50.

6. Yates P, Zhao I. Update on complex nonpharmacological interventions for breathlessness. Curr Opin Support Palliat Care 2012;6:144-52.

7. Di Filippo ES, Mancinelli R, Marrone M, et al. Neuromuscular electrical stimulation improves skeletal muscle regeneration through satellite cell fusion with myofibers in healthy elderly subjects. J Appl Physiol (1985) 2017;123:501-12.
8. Brüggemann AK, Mello CL, Dal Pont T, et al. Effects of Neuromuscular Electrical Stimulation During Hemodialysis on Peripheral Muscle Strength and Exercise Capacity: A Randomized Clinical Trial. Arch Phys Med Rehabil 2017;98:822-831.e1.

9. Maddocks M, Nolan CM, Man WD, et al. Neuromuscular electrical stimulation to improve exercise capacity in patients with severe COPD: a randomised double-blind, placebo-controlled trial. Lancet Respir Med 2016;4:27-36.

10. Nozoe M, Kanai M, Kubo H, et al. Efficacy of neuromuscular electrical stimulation for preventing quadriceps muscle wasting in patients with moderate or severe acute stroke: A pilot study. NeuroRehabilitation 2017;41:143-9.

11. Bonnevie T, Gravier FE, Debeaumont D, et al. Homebased neuromuscular electrical stimulation as an add-on to pulmonary rehabilitation does not provide further benefits in patients with chronic obstructive pulmonary disease: A multicenter randomized trial. Arch Phys Med Rehabil 2018;99:1462-70.

12. Pan L, Guo Y, Liu X, et al. Lack of efficacy of neuromuscular electrical stimulation of the lower limbs in chronic obstructive pulmonary disease patients: a metaanalysis. Respirology 2014;19:22-9.

13. Jadad AR, Moore RA, Carroll D, et al. Assessing the quality of reports of randomized clinical trials: is blinding necessary? Control Clin Trials 1996;17:1-12.

14. Kjaergard LL, Villumsen J, Gluud C. Reported methodologic quality and discrepancies between large and small randomized trials in meta-analyses. Ann Intern Med 2001;135:982-9.

15. Liberati A, Altman DG, Tetzlaff J, et al. The PRISMA statement for reporting systematic reviews and metaanalyses of studies that evaluate healthcare interventions: explanation and elaboration. BMJ 2009;339:b2700.

16. Higgins JP, Thompson SG, Deeks JJ, et al. Measuring inconsistency in meta-analyses. BMJ 2003;327:557-60.

17. Bourjeily-Habr G, Rochester CL, Palermo, F. et al. Randomised controlled trial of transcutaneous electrical muscle stimulation of the lower extremities in patients with chronic obstructive pulmonary disease. Thorax 2002;57:1045-9.

18. Maddocks M, Lewis M, Chauhan A, et al. Randomized controlled pilot study of neuromuscular electrical stimulation of the quadriceps in patients with non-small cell lung cancer. J Pain Symptom Manage 2009;38:950-6.

19. Maddocks M, Halliday V, Chauhan A, et al.

Neuromuscular electrical stimulation of the quadriceps in 
patients with non-small cell lung cancer receiving palliative chemotherapy: a randomized phase II study. PLoS One 2013;8:e86059.

20. Neder JA, Sword D, Ward SA, et al.Home based neuromuscular electrical stimulation as a new rehabilitative strategy for severely disabled patients with chronic obstructive pulmonary disease (COPD). Thorax 2002;57:333-7.

21. Vivodtzev I, Pepin JL, Vottero G, et al. Improvement in quadriceps strength and dyspnea in daily tasks after 1 month of electrical stimulation in severely deconditioned and malnourished COPD. Chest 2006;129:1540-8.

22. Vivodtzev I, Debigare R, Gagnon P, et al. Functional and muscular effects of neuromuscular electrical stimulation in patients with severe COPD: a randomized clinical trial. Chest 2012;141:716-25.

23. Vivodtzev I, Decorte N, Wuyam B, et al. Benefits of neuromuscular electrical stimulation prior to endurance training in patients with cystic fibrosis and severe pulmonary dysfunction. Chest 2013;143:485-93.

24. Sillen MJ, Franssen FM, Delbressine JM, et al. Efficacy of lower-limb muscle training modalities in severely dyspnoeic individuals with COPD and quadriceps muscle weakness: results from the DICES trial. Thorax 2014;69:525-31.

25. Vieira PJ, Chiappa AM, Cipriano G, et al. Neuromuscular electrical stimulation improves clinical and physiological function in COPD patients. Respir Med 2014;108:609-20.

26. Tasdemir F, Inal-Ince D, Ergun P, et al. Neuromuscular electrical stimulation as an adjunct to endurance and resistance training during pulmonary rehabilitation in stable chronic obstructive pulmonary disease. Expert Rev

Cite this article as: Gong H, Jiang Q, Shen D, Gao J. Neuromuscular electrical stimulation improves exercise capacity in adult patients with chronic lung disease: a meta-analysis of English studies. J Thorac Dis 2018;10(12):6722-6732. doi: 10.21037/jtd.2018.11.133
Respir Med 2015;9:493-502.

27. Dowman LM, McDonald CF, Hill CJ, et al. The evidence of benefits of exercise training in interstitial lung disease: a randomised controlled trial. Thorax 2017;72:610-9.

28. Güell MR, Cejudo P, Ortega F, et al. Benefits of LongTerm Pulmonary Rehabilitation Maintenance Program in Patients with Severe Chronic Obstructive Pulmonary Disease. Three-Year Follow-up. Am J Respir Crit Care Med 2017;195:622-9.

29. Henoch I, Strang S, Lofdahl CG, et al. Health-related quality of life in a nationwide cohort of patients with COPD related to other characteristics. Eur Clin Respir J 2016;3:31459.

30. Puhan MA, Chandra D, Mosenifar Z, et al. The minimal important difference of exercise tests in severe COPD. Eur Respir J 2011;37:784-90.

31. Wouters EF. Minimal clinically important differences in COPD: body mass index and muscle strength. COPD 2005;2:149-55.

32. Vivodtzev I, Lacasse Y, Maltais F. Neuromuscular electrical stimulation of the lower limbs in patients with chronic obstructive pulmonary disease. J Cardiopulm Rehabil Prev 2008;28:79-91.

33. Sillen MJH, Speksnijder CM, Eterman RA, et al. Effects of neuromuscular electrical stimulation of muscles of ambulation in patients with chronic heart failure or COPD: a systematic review of the English-language literature. Chest 2009;136:44-61.

34. Chen RC, Li XY, Guan LL, et al. Effectiveness of neuromuscular electrical stimulation for the rehabilitation of moderate-to-severe COPD: a meta-analysis. Int J Chron Obstruct Pulmon Dis 2016;11:2965-75. 